\title{
Employment of Some Parameters to Enhance Laser-Drilling of Aluminum
}

\author{
Oday A. Hamadi
}

P.O. Box 55159, Baghdad 12001, IRAQ, Email:odayata2001@yahoo.com.

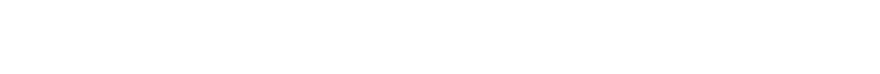

عهي علاحماي

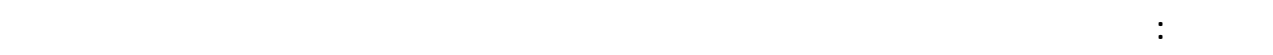

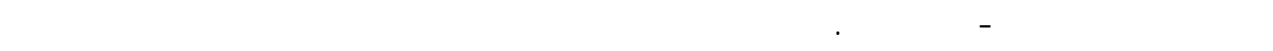

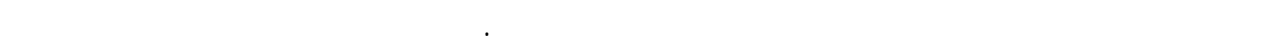

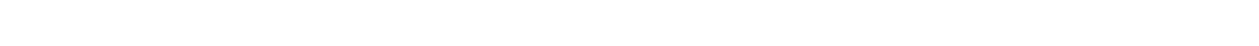

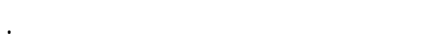

\begin{abstract}
In this work, some parameters affecting drilling of aluminum samples by a pulsed Nd:YAG laser were studied. These parameters are multi-pulses irradiation, controlling sample temperature, low-pressure ambient and application of electric field on the sample. Results presented in this work explained that these parameters can enhance drilling process throughout increasing hole depth in aluminum samples at the same laser energy used for irradiation.
\end{abstract}

KEYWORDS: Laser, aluminum and aplication

\section{Introduction}

$\mathbf{L}$

aser-material interaction is determined mainly by reflection, absorption and transmission of the material to the wavelength of laser beam. Laser beam is partially reflected at the surface of material and this reflection represents a loss in the incident energy and hence energy contributing the interaction. Another part of incident energy is absorbed since transmission is supposed to be neglected in metals. The absorbed energy may be dissipated due to thermal conduction of the material as well as absorption and scattering of laser beam by removed or evaporated material during the process (Ready 1978a).

Laser heating of opaque metals is a surface effect. This leads to a rise in temperature of the metal surface since the energy absorption is higher at the surface and decreasing with depth inside the material due to the effect of thermal diffusivity of the material (Wagner 1974). The following relation determines the rise in surface temperature caused by laser irradiation as (Wilson and Hawkes 1987): 


$$
T_{S}=T_{\mathrm{o}}+2 I_{\mathrm{o}} \frac{1-R}{k}\left(\frac{N \tau}{\pi}\right)^{\frac{1}{2}}
$$

where $T_{S}$ and $T_{0}$ are the final measured and initial temperatures of the surface, respectively, $I_{o}$ is the incident power density, $R$ is the surface reflectivity, $k$ is thermal conductivity, $N$ is thermal diffusivity and $\tau$ is the laser pulse duration.

Laser irradiation is an important source to heat the material during a very short time to high temperatures that cannot be achieved by conventional sources. Metal temperature varies with time at a rate of $10^{6} \mathrm{C} / \mathrm{s}$ during laser-material interaction in drilling (von Allmen 1978). Regarding equation (1), the surface temperature is related to the square root of the laser pulse duration $\tau$ as well as thermal diffusivity $N$, and related inversely to the thermal conductivity $k$. The latter has a negative effect on surface temperature since the higher thermal conductivity admits more thermal energy to dissipate inside the material. In turn, the surface temperature in laser-material interaction relates to its reflectivity $R$. Practically, surface reflectivity decreases during irradiation time and is not constant as seen in equation (1) (Ready 1978b, Chun and Rose 1970).

Vaporization and material removal are the principles of cutting and drilling processes by laser. When the laser power density is as high as $10^{6} \mathrm{~W} / \mathrm{cm}^{2}$, surface temperature rises fast to the vaporization point. Accordingly, there will be no more melting as vaporization is reached and a hole is formed.

Interaction between a laser beam and a metal surface is dependent on several parameters, such as incident power density, pulse duration and surface absorption. Consequently laser drilling and material removal are dependent on these parameters.

Laser drilling is one of the earliest applications that accompanied laser invention. Hence, it attracted a wide interest by researchers and its fundamentals are now well established. About $90 \%$ of the material removed in laser drilling is in the form of liquid drops condensed on the walls of the hole as the rest being material vapor (Ready 1978a, Charchan 1972). The velocity of material removal and the hole formation are related by (Steen 1991):

$$
v_{s}=\frac{I}{\rho\left[c\left(T_{v}-T_{0}\right)+L_{v}\right]}
$$

where $I$ is the absorbed power density, $\rho$ is the material density, $c$ is the specific heat capacity, $T_{v}$ and $L_{v}$ are temperature and latent heat of vaporization, respectively. The depth of the hole formed by a laser pulse is given by (Luxon and Parker 1985):

$$
d=\frac{E_{0}}{a \rho\left[c\left(T_{v}-T_{0}\right)+L_{v}\right]}
$$

where $E_{0}$ is the incident laser energy and $a$ is the area affected by laser beam.

When the absorption of the incident laser power and the rise in surface temperature to vaporization point occurr, three consequent processes result in hole formation and material removal; they are (Chun and Rose 1970):

a) Surface vaporization during the stage of laser pulse

b) Rise in pressure inside the crater formed due to the removal of the surface layer as a result of vapor expansion

c) Removal of the melt outside the hole.

There are many advantages of the laser drilling compared to the conventional methods. Contamination of the processed region is already avoided since the process is carried out by a light (laser) beam, i.e., contactless processing. High accuracy drilling, small size holes and hence small and limited heat affected zone (HAZ) and invariant properties of material are satisfied by laser. Brittle 


\section{EMPLOYMENT OF SOME PARAMATERS}

materials such as glass and ceramics can be drilled by laser more easily than by conventional methods. Also, some solid materials that cannot be drilled or processed conventionally, are drilled easily by high power lasers of high power densities and short pulses. Thus, laser drilling is the fastest since processing time is as short as laser pulse duration.

Laser drilling has some disadvantages, due to the limited transmission of laser beam inside material and the limited penetration. Internal surface of hole is rather non-uniform and rough due to condensation of the material vapor. Somehow, laser drilling is so expensive compared to the conventional methods.

\section{Experiment}

Samples of high-pure aluminum were cut with $1 \times 1 \mathrm{~cm}^{2}$ area and $3 \mathrm{~mm}$ thickness, polished, grinded, distilled in water and alcohol and then dried with hot air. Samples were irradiated by a pulsed JK2000 Nd:YAG laser of $1.06 \mu \mathrm{m}$ wavelength, 7J maximum output energy and $300 \mu \mathrm{s}$ pulse duration. The maximum laser power density obtained was $4.8 \mathrm{MW} / \mathrm{cm}^{2}$. Dimensions of the holes in the samples were introduced and measured by a Leitz-Metallux 3 optical microscope. Samples were moved in front of laser beam by an $x-y$ micrometer stage.

In order to introduce the effect of sample preheating on laser drilling, a tungsten lamp of 650W power and 0.5-3.5 $\mu \mathrm{m}$ emission spectrum to heat the sample and a K-type thermocouple to measure temperature were used. A voltage supply was used to apply an electric field on the sample mounted between two parallel 1cm-spaced copper plates. To study the effect of ambient pressure on the drilling process, a vacuum chamber as down as $10^{-2}$ mbar with a rotary vacuum pump was used. The data were fitted by a 2-order polynomial function to explain the best behavior.

\section{Results and Discussion}

In Figure 1, the hole depth is measured with laser energy and it was observed that the hole depth increased as irradiation laser energy was increased. This increase in the laser energy caused the material to absorb more energy and raise the surface temperature of the sample. The amount of material melted and removed from the hole increased too due to the vapor pressure generated (Ready 1978a; Chun and Rose 1970). At higher energies, the variation of the hole depth is not linear with the variation of laser energy because the energy absorbed by the material - that should increase with increasing incident laser energy is affected by vapor and plasma formed and may absorb some energy (Ready 1978b; Duley 1987; Nonhof 1988). The results in Figure 1 are obtained by a single pulse irradiation.

Samples were then irradiated by multi-pulses of laser with a $100 \%$ overlap. The whole thickness of the aluminum sample was penetrated by 6 pulses as shown in Figure 2. The effect of multi-pulses irradiation is interpreted as being due to two reasons. First, the variation of the laser beam focus just after the first laser pulse is due to vaporization of a layer of material and formation of the hole. Now, the laser beam is not focused at the bottom of hole and this increases the spot size and decreases the laser power density, hence causing the vaporization and the material removal by the next pulse and so on (Rykalin, et al. 1978). The second reason is that the phase transformation at the bottom of the hole due to the vaporization and the material removal by the first laser pulse causes heat accumulation. Resolidification of some melted material on the walls of the hole may distort the hole section and decrease material removal by the next pulse. This decreases the effect of the next pulse (Banas and Webb 1982). In general, despite the fact that material removal decreases after the first pulse, the hole depth increases with increasing number of pulses on the hole as each pulse removes a layer of material (Ready 1978a, Ready 1978b). 


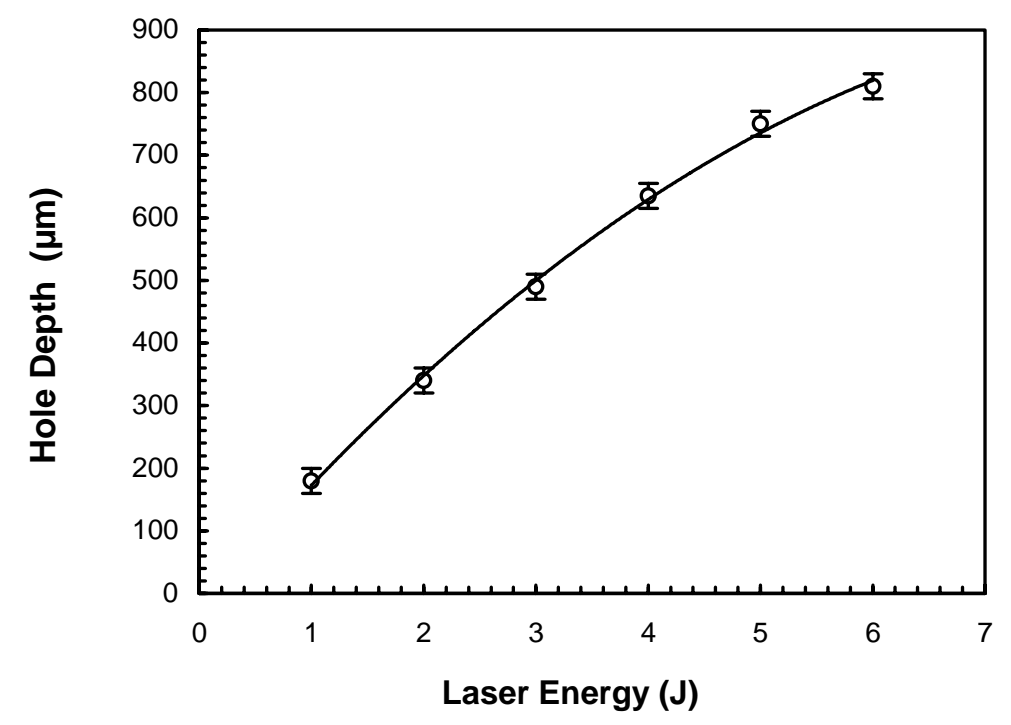

Figure 1. Variation of the hole depth measured with varying incident laser energy.

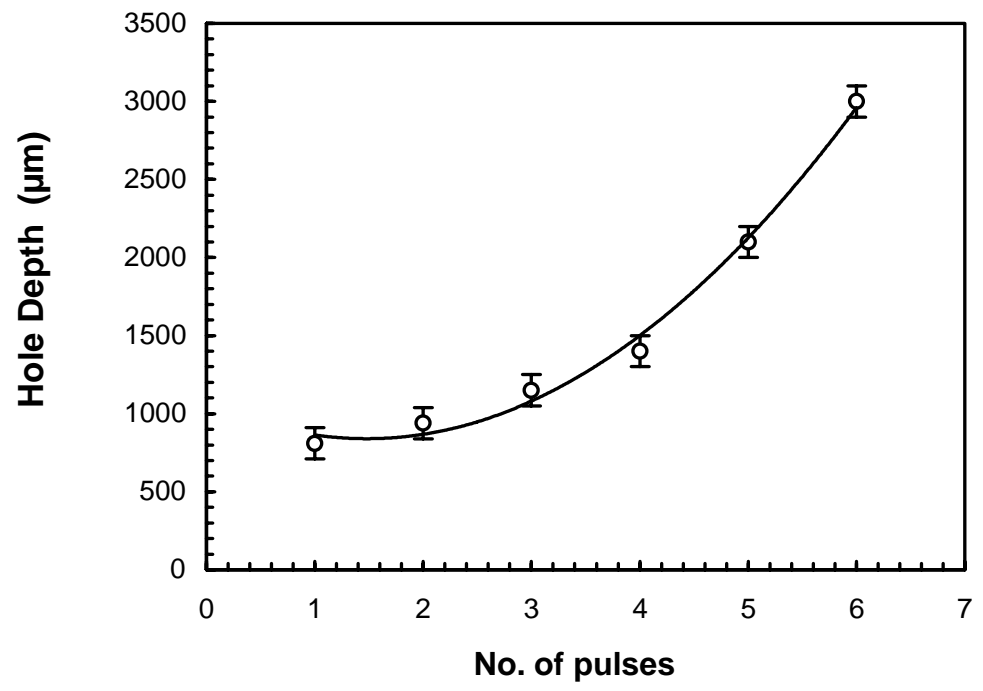

Figure 2. Effect of multi-pulses irradiation on the variation of the hole depth measured with varying incident laser energy $(\mathrm{E}=6 \mathrm{~J})$. 


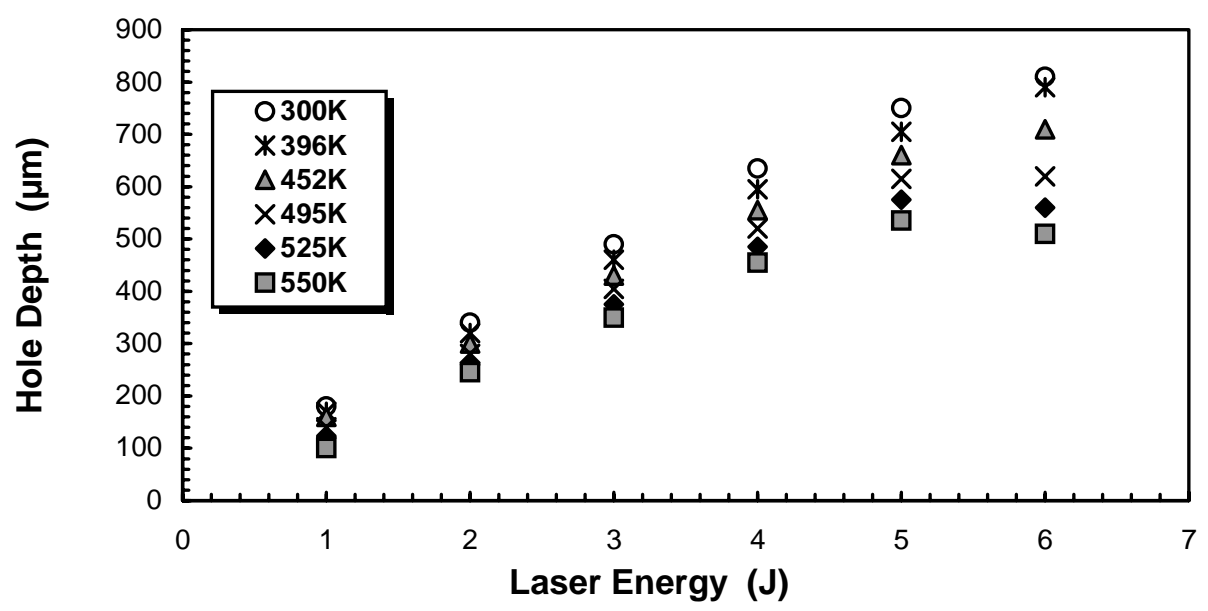

Figure 3. The effect of sample preheating on the variation of the hole depth measured with varying incident laser energy.

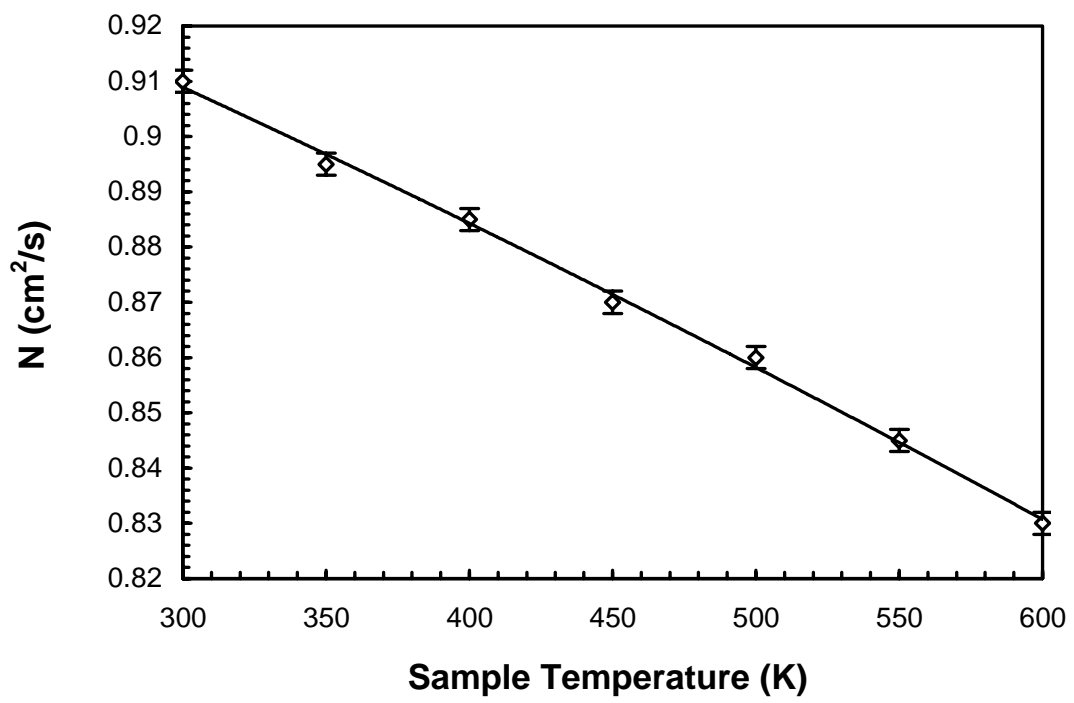

Figure 4. The behavior of thermal diffusivity of the aluminum sample with temperature. 


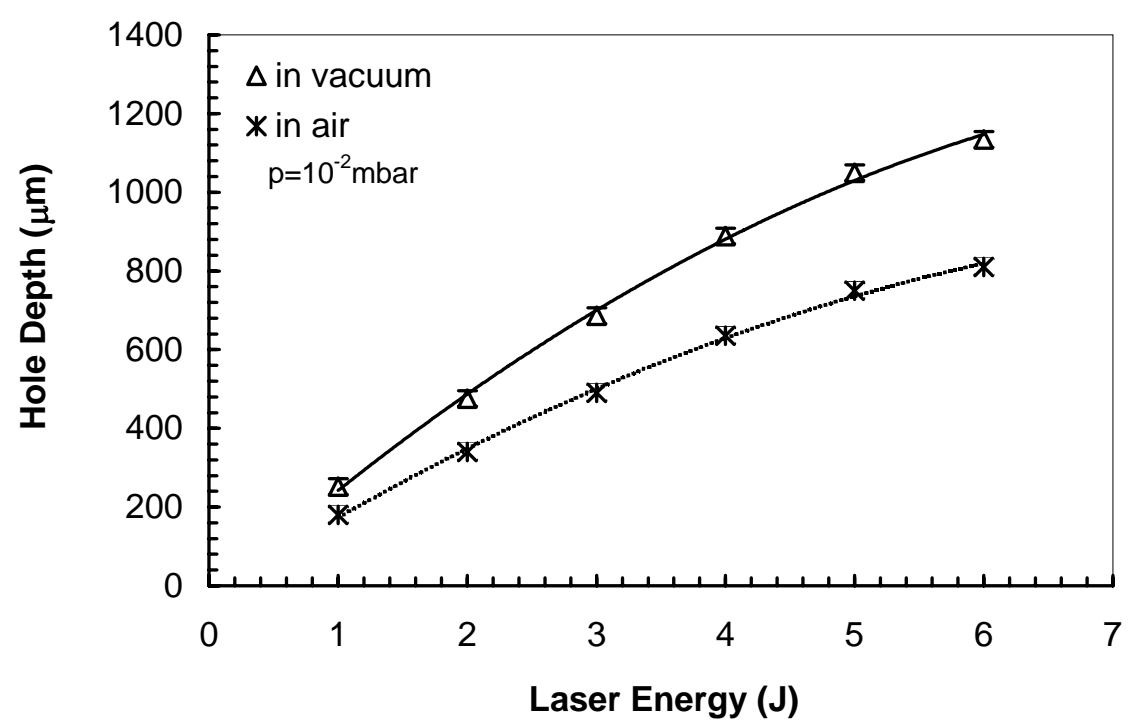

Figure 5. The effect of environment vacuum on the variation of the hole depth measured with varying incident laser energy.

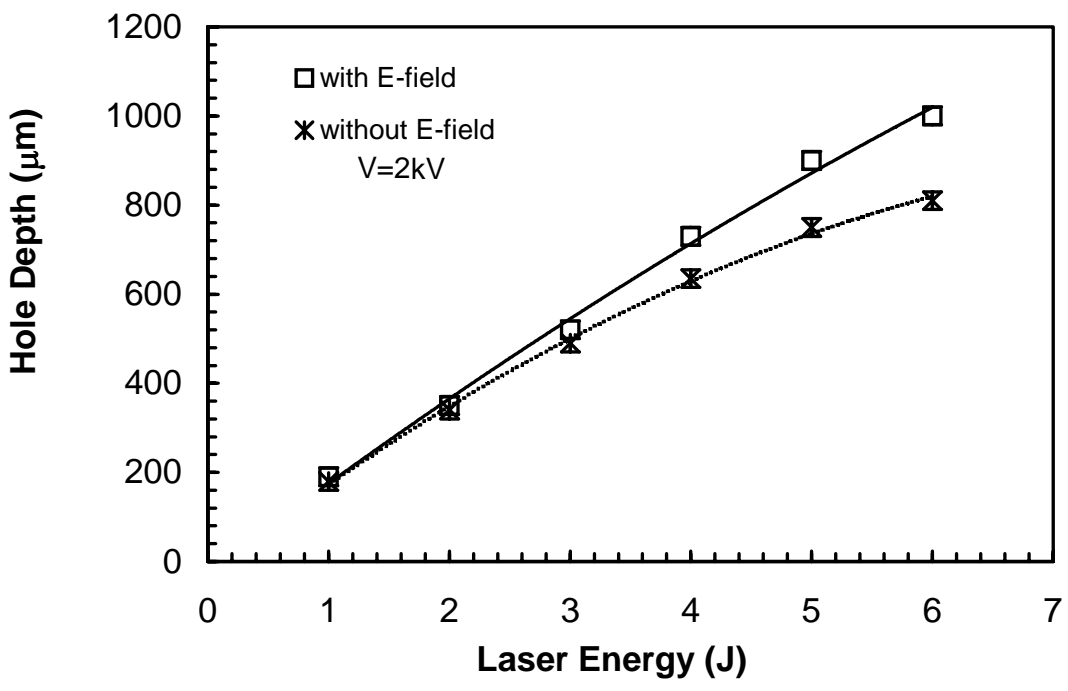

Figure 6. The effect of applied electric field on the variation of the hole depth measured with varying incident laser energy. 


\section{EMPLOYMENT OF SOME PARAMATERS}

Figure 3 indicates the effect of the sample preheating on the hole depth, as can be seen, all curves of different preheating temperatures are similar to the case of non-heating (300K) but with a decrease in the hole depth. This is attributed to the effect of thermal diffusivity which drops with temperature contrary to the case in most metals. Figure 4 shows the behavior of thermal diffusivity of an aluminum sample with temperature. This effect shows that it is better to carry out drilling of aluminum at room temperature. Samples were mounted and irradiated inside the vacuum chamber at a $10^{-2}$ mbar pressure and the hole depth was measured as a function of incident laser energy, then compared to results obtained in air. Figure 5 explains the effect of vacuum environment on laser drilling; the hole depth increases by about $40 \%$ in vacuum than in air. Low pressure ambient contributes effectively to the vapor expansion and the material removal (Rykalin, et al. 1978) and hence increases the laser beam transmission inside the hole leading to more penetration inside the sample (von Allmen 1976).

The effect of the electric field on laser drilling of the aluminum sample is also studied. Figure 6 shows the hole depth measured as a function of laser energy. The hole depth increases by $23 \%$ due to application of the electric field compared to the case without applied field. This effect may be caused by the force resulting from the applied electric field. Since material vapor contains charged particles (electrons and ions), this force removes these particles from the hole decreasing the absorption of the laser beam energy by the vapor and hence increasing the penetration depth inside the sample (Chen et al. 1996, Voisey et al. 1999, Lehane and Kwok 2001).

\section{Conclusions}

According to the results obtained and presented in this work, drilling of an aluminum samples by pulsed Nd:YAG laser can be controlled and enhanced employing several parameters. Multi-pulses irradiation, control of sample temperature, low-pressure environment and application of electric field can enhance the process and increase the hole depth. Employing all four parameters, the hole depth can be increased to twice its expected size. With any doubt, the results obtained in this work could not have been achieved without employing these parameters for the same laser energy used for the irradiation, thus the improvement advantages to laser drilling processes.

\section{References}

BANAS, C. and WEBB, R. 1982. Laser Macro-Material Processing. Proc. IEEE. 70(6): 556.

CHARCHAN, S. 1972. Lasers in Industry. Van Nostrand Reinhold Co., Ch.3, Ch.4, pp.247-256.

CHEN, X., LOTSHAW, W.T., ORTIZ, A.L., STAVER, P.R., ERIKSON, C.E., MCLAUGHLIN, M.H. and ROCKSTROH, T.J. 1996. Laser drilling of advanced aterials: effects of peak power, pulse format and wavelength. J. Laser Appl. 8(5): 233-239.

DULEY, W. 1976. $\mathrm{CO}_{2}$ Lasers Effects and Applications, Ch. 4,5 Academic Press , New York.

LEHANE, C. 1976 and KWOK, H.S. 2001. Enhanced drilling using a dual-pulse Nd:YAG laser. Appl. Phys. A 73: 45-48.

LUXON, J. and PARKER, D. 1985. Industrial Lasers and Their Applications. Ch. 11, Prentice-Hall.

NONHOF, C. 1988. Material Processing with Nd-Lasers, Ch. 1,5,6, Electro-chemical Publications Ltd.

READY, J. 1978. Industrial Applications of Lasers. Academic Press, Ch. 13-15.

READY, J. 1978. Lasers in Modern Industry. Dearbon (Michigan), p.73, 203.

RYKALIN, N, UGLOV, A. and KOKORA, A. 1978. Laser Machining and Welding. Pergamon Press, Ch. 1, 2, 4.

STEEN, W. 1991. Laser Material Processing. p.74, 75, 99, 101.

VOISEY, K.T., WESTLEY, J., BYRD, P. and CLYNE, T.W. 1999. Effects of assist gas in the laser drilling of thermal barrier coated superalloys, Online information. 
VON ALLMEN, M., BLASÉ, P., AFFOLTER, K. and STRUMER, E. 1978. Absorption phenomena in metal drilling with Nd-lasers. IEEE J. Quantum Electron. 14(2): 85-88.

VON ALLMEN, 1976. Laser drilling velocity in metals. J. Appl. Phys. 47(12): 5460-5463.

WILSON, J. and A. HAWKES, J. 1987. Lasers Principles and Applications. Ch. 5, Prentice-Hall.

Received 16 May 2004

Accepted 13 February 2005 